\title{
Conductance switching in a molecular device: The role of side groups and intermolecular interactions
}

\author{
Taylor, Jeremy Philip; Brandbyge, Mads; Stokbro, Kurt
}

Published in:

Physical Review B Condensed Matter

Link to article, DOI:

10.1103/PhysRevB.68.121101

Publication date:

2003

Document Version

Publisher's PDF, also known as Version of record

Link back to DTU Orbit

Citation (APA):

Taylor, J. P., Brandbyge, M., \& Stokbro, K. (2003). Conductance switching in a molecular device: The role of side groups and intermolecular interactions. Physical Review B Condensed Matter, 68(12), 121101. https://doi.org/10.1103/PhysRevB.68.121101

\section{General rights}

Copyright and moral rights for the publications made accessible in the public portal are retained by the authors and/or other copyright owners and it is a condition of accessing publications that users recognise and abide by the legal requirements associated with these rights.

- Users may download and print one copy of any publication from the public portal for the purpose of private study or research.

- You may not further distribute the material or use it for any profit-making activity or commercial gain

- You may freely distribute the URL identifying the publication in the public portal 


\title{
Conductance switching in a molecular device: The role of side groups and intermolecular interactions
}

\author{
Jeremy Taylor, Mads Brandbyge, and Kurt Stokbro \\ Mikroelektronik Centret (MIC), Technical University of Denmark (DTU), Building 345 east, DK-2800 Kongens Lyngby, Denmark
}

(Received 16 June 2003; published 12 September 2003)

\begin{abstract}
We report first-principles studies of electronic transport in monolayers of Tour wires functionalized with different side groups. An analysis of the scattering states and transmission eigenchannels suggests that the functionalization does not strongly affect the resonances responsible for current flow through the monolayer. However, functionalization has a significant effect on the interactions within the monolayer, so that monolayers with $\mathrm{NO}_{2}$ side groups exhibit local minima associated with twisted conformations of the molecules. We use our results to interpret observations of negative differential resistance and molecular memory in monolayers of $\mathrm{NO}_{2}$ functionalized molecules in terms of a twisting of the central ring induced by an applied bias potential.
\end{abstract}

DOI: 10.1103/PhysRevB.68.121101

PACS number(s): 71.15.Mb, 73.63.-b, 85.65.+h

An important goal in the study of molecular electronics is to identify molecules that can be combined to perform logical functions. ${ }^{1}$ In particular, Reed and co-workers ${ }^{2-5}$ have studied the electrical properties of a set of phenyl-ethylene oligomers [known as Tour wires (TW's) ${ }^{6}$ ] functionalized with different side groups, and demonstrated that such molecules can show negative differential resistance (NDR) ${ }^{2,3}$ and a "molecular memory", 4,5 effect, in which case molecules can be switched from a low conductance state to a high conductance state by application of a voltage pulse. A fundamental understanding of the microscopic mechanisms governing the NDR and memory effects in these systems is still lacking.

Complementary studies, by Weiss and co-workers, ${ }^{7}$ of isolated or small bundles of TW's embedded in a monolayer of spacer alkyl molecules, demonstrated spontaneous conductance switching. While Reed and co-workers only observed switching effects for TW's with $\mathrm{NO}_{2}$ side groups, Weiss and co-workers reported spontaneous switching of all the molecules in their study but had limited success in inducing switching by using a voltage pulse. ${ }^{7}$

Theoretical studies of functionalized TW's, have so far been restricted to isolated molecules. Based on such studies, Seminario et al. proposed that charging of the molecule and subsequent localization/delocalization of molecular orbitals is the microscopic mechanism behind NDR, ${ }^{8,9}$ while Cornil et al. have found that bias-induced alignment of molecular orbitals on the first and last phenyl rings of twisted TW's can lead to NDR. ${ }^{10}$

In this paper we present the first studies of the electrical properties of functionalized TW's covalently bound to gold surfaces. Most prominent previous studies of related systems include the $I V$ characteristics of a functionalized benzene ring $^{13}$ and a bare TW. ${ }^{14}$ Our calculations are based on the TRANSIESTA package, ${ }^{11}$ which is a nonequilibrium Greensfunction-based electronic transport program founded on density-functional theory. ${ }^{12}$ It treats the entire system selfconsistently under finite bias conditions.

The nanopore structures in which NDR and memory effects have been observed are formed by evaporating the molecules onto a small $\mathrm{Au}$ surface inside a $\mathrm{Si}_{3} \mathrm{~N}_{4}$ structure, and then evaporating another $\mathrm{Au}$ electrode onto the molecular monolayer. ${ }^{2}$ There is no detailed information about the structure of the monolayer, and the purpose of this study is not an accurate numerical model of the nanopore experiment. We will form an idealized model of an electrode/monolayer/ electrode system and use the insight from our studies to make general statements about the effect of functionalization on the electron-transport properties of TW's. We will show that while DeVentra et al. found intrinsic NDR for a functionalized benzene ring, the ground-state geometry of a functionalized TW does not show any NDR, and has an $I V$ characteristics similar to the unfunctionalized TW. Instead, our calculations show that functionalization stabilizes twisted conformations within the monolayer, and we propose that NDR and memory effects are related to such conformational changes.

We use an $\mathrm{Au}(111)$ surface in a $3 \times 3$ unit cell for the electrodes and assume that the TW's are chemisorbed to the surfaces through strong thiol bonds, ${ }^{15}$ as illustrated in Fig. 1. Monolayer $A$ consists of a bare TW with thiol end groups, while monolayers $B$ and $C$ have been functionalized with $\mathrm{NH}_{2}$ and $\mathrm{NO}_{2}$ side groups, respectively.

We first investigate the effect of the side groups on the electrical properties of the monolayers. The current through the monolayers is determined by the quantum-mechanical probability for electrons to tunnel from one electrode to the other, and is calculated using the Landauer formula ${ }^{16} I$ $=\int_{\mu_{L}}^{\mu_{R}} T\left(E, V_{b}\right) d E$, where $\mu_{L / R}= \pm e V_{b}$ and $T\left(E, V_{b}\right)$ is the transmission probability for electrons incident at an energy $E$ through a device under a potential bias $V_{b}$ (n.b $V_{b}>0$ corresponds to hole injection from the right electrode). The general shape of the zero-bias transmission spectra, shown as $T\left(E, V_{b}=0 V\right)$ in Fig. 2(a), is similar for all three systems. The zero-bias conductance $G=\left(e^{2} / h\right) T\left(E=\mu_{l / r}, V_{b}=0\right)$, given by $2.0 \mu \mathrm{S}, 2.3 \mu \mathrm{S}$, and $1.9 \mu \mathrm{S}$, for monolayers $A, B$, and $C$, respectively, is dominated by the tail of a broad highest-occupied-molecular-orbital (HOMO) resonance (at $E_{\mathrm{HOMO}}=-0.84,-0.65$, and $-0.85 \mathrm{eV}$ for monolayers $A$, $B$, and $C$ ). The narrower lowest-unoccupied-molecularorbital (LUMO) resonance (at $E_{\mathrm{LUMO}}=1.56,1.59$, and $1.14 \mathrm{eV}$ for $A, B$, and $C$ respectively) contributes less to the conductance, and thus holes are the dominant carriers. The 


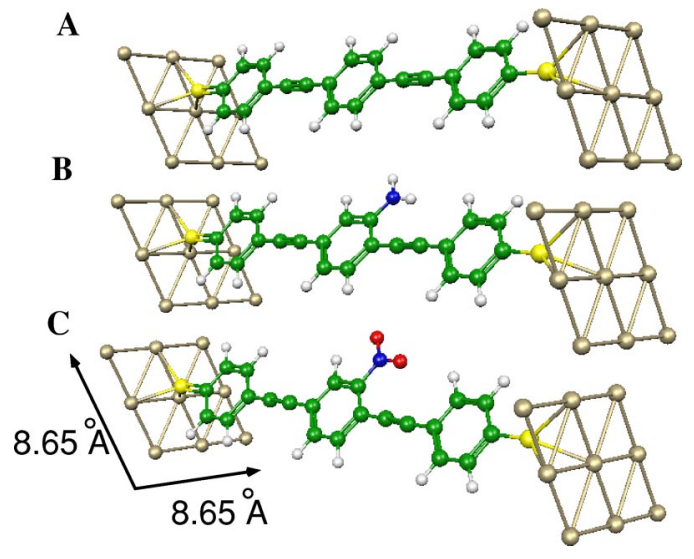

FIG. 1. (Color online) Geometry of monolayers A-C connected with two $\mathrm{Au}$ (111) surfaces. Color codes: C (dark gray or green), $\mathrm{H}$ (white), O (black or red), N (black or blue), S (light gray or yellow), and Au (light gray or gold).

$\mathrm{NH}_{2}$ group is electron donating, so that $E_{\mathrm{HOMO}, B}-E_{\mathrm{HOMO}, A}$ $\approx 200 \mathrm{meV}$ while the $\mathrm{NO}_{2}$ group is electron accepting so $E_{\mathrm{LUMO}, C}-E_{\mathrm{LUMO}, A} \approx-400 \mathrm{meV}$.

To calculate the $I-V_{b}$ spectrum we performed selfconsistent calculations for biases in the range -2.8 to $2.8 \mathrm{~V}$ in steps of $0.2 \mathrm{~V}$. We note that the charge on the molecule is not fixed $^{11}$ and adjusts itself to minimize the free energy ${ }^{17}$ as the left/right electrochemical potentials are changed. For all three systems we find that the molecules are close to charge neutrality, and the charge on the functionalized molecules $Q_{M}$, as determined by a Mulliken population analysis, change by less than $0.03 e$ as the bias voltage $V_{b}$ is varied. ${ }^{18}$ The $I-V_{b}$ spectra, shown in Fig. 2(b), are very similar, increasing slowly at first but then increasing rapidly around $2 \mathrm{~V}$ where the resonances come into alignment with the bias window. The main effect of $V_{b}$ on $T\left(E, V_{b}\right)$ is to sample more and more of the resonance while resonant peak height and position vary slowly with $V_{b} \cdot{ }^{14}$

When the molecules interact with the $\mathrm{Au}(111)$ electrodes, the molecular levels broaden into a continuum. ${ }^{16}$ The eigenstates of the whole metal/monolayer/metal system consist of scattering states, ${ }^{19,20}$ which are molecular-orbital-like in the molecule, and Bloch-wave-like in the metal slabs. If an orbital is delocalized across the molecule, an electron that enters the molecule at the energy of the orbital has a high probability of reaching the other end, and thus there is a corresponding peak in the transmission probability $T\left(E, V_{b}\right)$, as illustrated in Fig. 2(a). By calculating the continuum eigenstates at the resonance energies $\left(E_{\mathrm{HOMO}}, E_{\mathrm{LUMO}}\right)$, the orbitals that are responsible for current flow through the molecule can be analyzed. The HOMO resonances, illustrated in Fig. 2(c), resemble the HOMO of the isolated molecules while the narrower LUMO resonances, illustrated in Fig. 2(d), resemble the LUMO of the isolated molecules. There are minor differences between the orbitals: the $\mathrm{NH}_{2}$ side group participates in the HOMO resonance but not in the LUMO resonance, while the $\mathrm{NO}_{2}$ group participates in the LUMO resonance and not the HOMO resonance, consistent with their respective donating/accepting characters. It is, however, clear from both the shape of the transmission
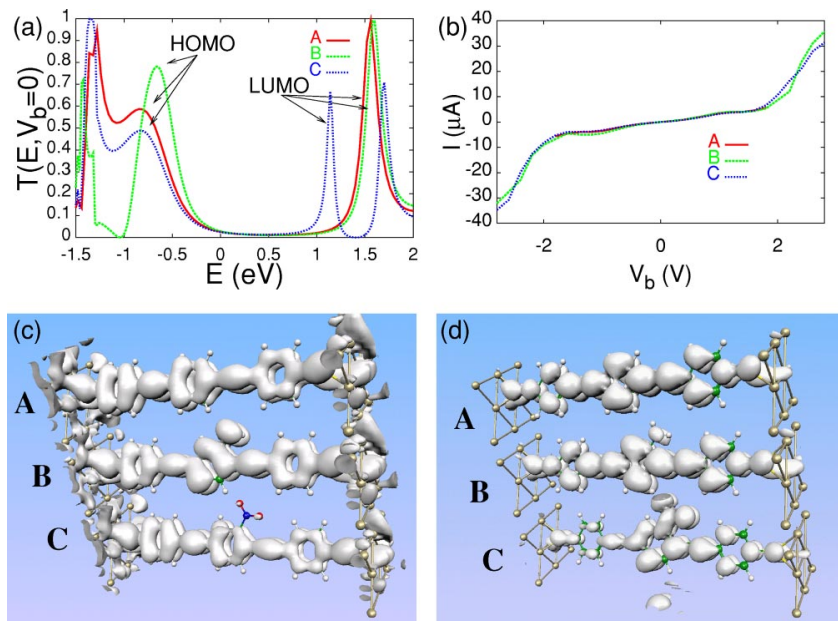

FIG. 2. (Color online) (a) Zero-bias transmission $T\left(E, V_{b}\right.$ $=0 \mathrm{~V}$ ) vs incident $E$ for monolayers $A, B$, and $C$. HOMO and LUMO resonances are indicated. (b) $I-V_{b}$ characteristics for monolayers $A, B$, and $C$. (c) Isosurface of transmission eigenchannel at the HOMO resonance for monolayers $A, B$, and $C(E=-0.84$, -0.65 , and $-0.85 \mathrm{eV}$, respectively) (d) Isosurface of transmission eigenchannel at the LUMO resonance for monolayers $A, B$, and $C$ ( $E=1.56,1.59$, and $1.14 \mathrm{eV}$ respectively).

curves in Fig. 2(a) and the orbitals in Figs. 2(c) and 2(d) that the resonant transmission peaks in monolayers $A, B$, and $C$ are related to the delocalized nature of the $\pi$ orbitals of the bare TW's and is not strongly affected by the functionalization.

The energetics of the monolayers are, however, strongly affected by the functionalization. The triple bond between the phenyl rings is rotationally symmetric and thus constitutes an easy axis about which the middle ring can rotate. The differences in total energy as a function of the rotation angle of the middle ring, $\theta$, for the molecules arranged in the $3 \times 3$ unit-cell of $\mathrm{Au}(111)$ are shown in Fig. 3(a). The intermolecular interaction energy is strongly dependent on the functionalization: there is a cost of $60 \mathrm{meV}(180 \mathrm{meV})$ to rotate the ring by $90^{\circ}$ in monolayer $A(B)$, and the flat molecule is clearly favored. Monolayer $C$ shows a quite different behavior, it acquires a local minimum at $\theta=60^{\circ}$ and $120^{\circ}$. Separate calculations show that if we double the distance between the molecules these minima disappear, thus they must be related to intermolecular interactions. Figure 3(b) shows the effective potential within the monolayer, and it reveals the formation of a hydrogen bond between the $\mathrm{NO}_{2}$ group and a hydrogen atom on the neighboring TW. Similar hydrogen bond formation involving $\mathrm{NO}_{2}$ groups has also been observed in other molecular layers. ${ }^{22}$ From Fig. 3(b) it is also clear that the minima at $60^{\circ}$ and $120^{\circ}$ can be attributed to bond formation with a new neighboring molecule, and these particular angles arise from the symmetry of the monolayer.

There is a barrier for rotation of the middle ring from $\theta$ $=0$ to $\theta=60^{\circ}$ of $\sim 150 \mathrm{meV}$, while the barrier from $60^{\circ}$ to $0^{\circ}$ is $\sim 65 \mathrm{meV}$. We postulate that, as a voltage pulse is applied, these barriers may change, and a transition to a new 


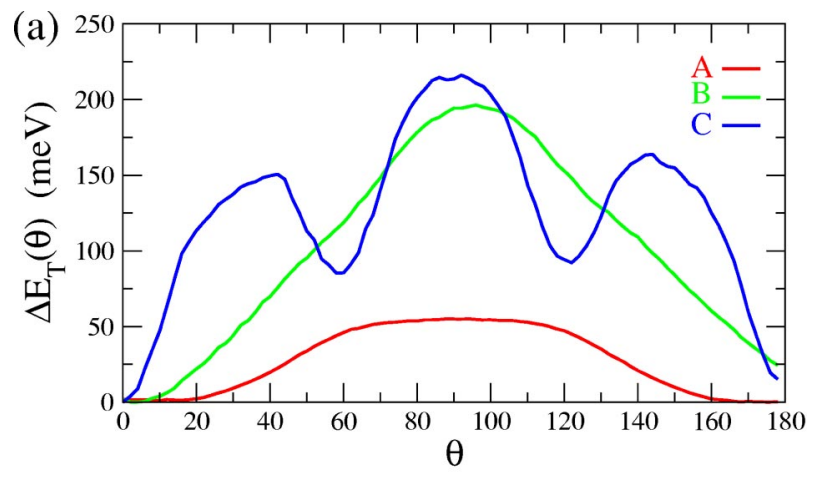

(b)

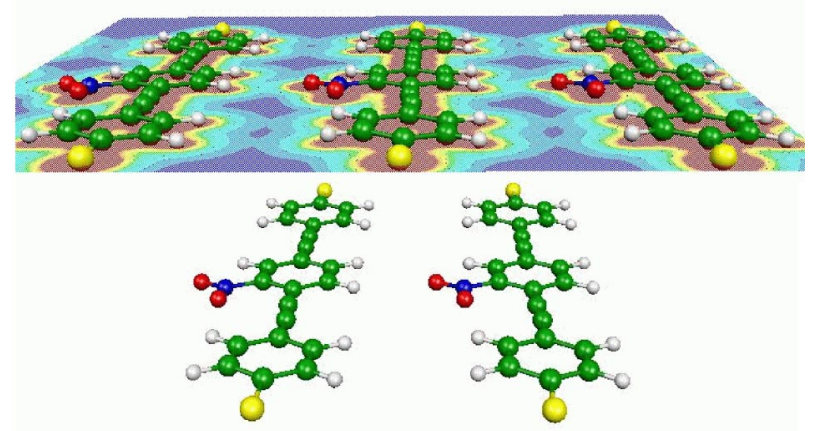

FIG. 3. (Color online) (a) Energy vs rotation angle for molecules $A, B$, and $C$ in $\mathrm{Au}(111) 3 \times 3$ unit-cell. The energy is calculated within the Perdew, Burke, and Ernzerhof approximation for the exchange-correlation functional (Ref. 21). (b) Contour plot of the effective potential between TW's with $\mathrm{NO}_{2}$ side groups. Note the bond formation between the $\mathrm{O}$ atom and the $\mathrm{H}$ atom on the neighboring TW.

molecular conformation with the middle ring twisted may be induced. We have selected a candidate conformation, which we designate monolayer C@60, formed by twisting the middle ring of molecules in monolayer $C$ by $60^{\circ}$, for possible stabilization under applied bias. The transmission spectra for monolayer C@60 is shown in Fig. 4(a) and its $I-V_{b}$ characteristics in Fig. 4(d). The conductance $G \approx 0.12 \mu \mathrm{S}$ is roughly 16 times smaller than monolayer $C$, and the current at $V_{b}=2 \mathrm{~V}$ is approximately five times smaller than in $C$. To lowest order, $G$ is proportional to the product of the matrix elements between $\pi$ orbitals on neighboring phenyl rings so that a rotation of the middle ring will reduce $G$ by a factor $\sim \cos ^{4}(\theta)=\frac{1}{8}$, in rough agreement with the calculated values. The transmission eigenstates for electrons traveling from left to right at $E_{\mathrm{HOMO}}=-0.85 \mathrm{eV}$ and $E_{\mathrm{LUMO}}=0.99 \mathrm{eV}$ are illustrated in Fig. 4(b). The HOMO resonance is localized on the phenyl ring nearest to the left electrode while the LUMO state corresponds to an orbital of the middle ring, weakly coupled to the first and last rings.

To investigate whether monolayer C@60 could be stabilized with respect to monolayer $C$ as the bias voltage is increased, we show in Fig. 4(b) the Kohn-Sham total energy of
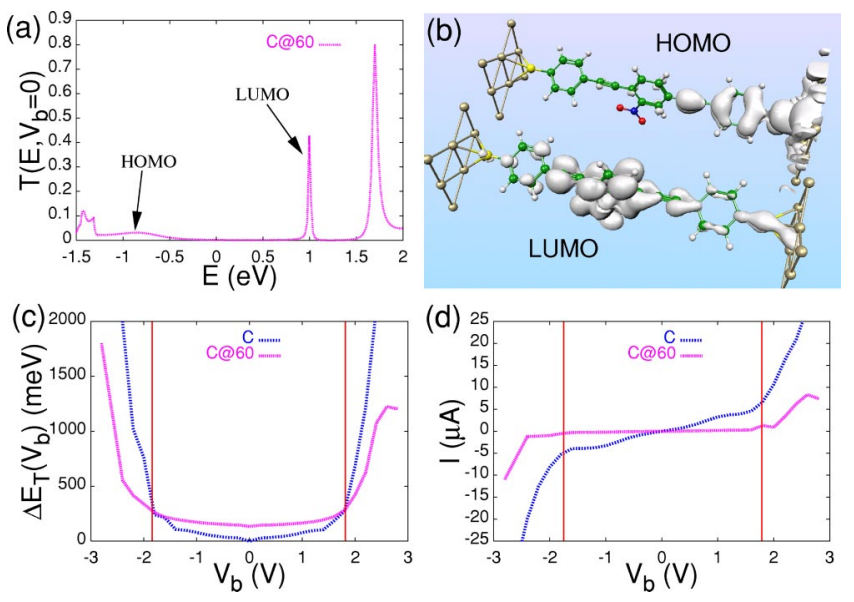

FIG. 4. (Color online) (a) Zero-bias transmission $T\left(E, V_{b}=0\right)$ for monolayer C@60. (b) Transmission eigenchannels corresponding to HOMO and LUMO resonances of C@60. (c) Total energy of monolayer a $\mathrm{C}$ (dark gray or blue) and monolayer C@60 (light gray or magenta) as a function of bias potential. (d) $I-V_{b}$ characteristics of monolayer C (dark gray or blue) and monolayer C@60 (light gray or magenta).

monolayers $C$ and C@60. At $V_{b}=0$, monolayer $C$ is lower in energy than monolayer C@60. However, when the bias is increased above $2 \mathrm{~V}$, monolayer C@60 becomes lower in energy, leading to an expected conformational change. While there are issues surrounding the evaluation of total energies for such systems, ${ }^{17,23}$ Fig. 4(b) serves to illustrate that different conformations may be stabilized with respect to each other by an external bias. In this case, the calculation suggests a transformation of monolayer $C$ to monolayer C@60 at around $2 \mathrm{~V}$. The exact value of the switching voltage will depend on the coverage, detailed structure, and size of the monolayer, as well as how the potential drops across the molecules, which can be affected by electrode coupling. ${ }^{14}$

The local minima in Fig. 3(a) are stabilized by the $\mathrm{NO}_{2}$ side group, which correlates well with that the molecular memory effect is only observed in monolayers with $\mathrm{NO}_{2}$ side groups. ${ }^{4}$ We find an energy barrier for rotation within an ideal $(3 \times 3)$ monolayer of $65-150 \mathrm{meV}$. This energy barrier will depend on the distance between the molecules in the monolayer, and we found that when doubling the distance between the molecules the barrier disappears. Most likely, the distance between the molecules in the nanopore experiments is smaller than for our ideal system, ${ }^{24}$ and since the energy barrier for the $(3 \times 3)$ monolayer is in rough agreement with the $80 \mathrm{meV}$ barrier height extracted from bit retention times, ${ }^{4}$ conformational changes stabilized by intermolecular interactions is a good candidate for explaining such phenomena.

Because the minima emerge from interactions within the monolayers, the above effects may only be observed in nanopore experiments $^{2-5}$ and not in experiments of single molecules. In the scanning tunneling microscopy experiments of Weiss and co-workers ${ }^{7}$ on single or small bundles of molecules, the conductance switching may be related to different 
conformations formed by steric interactions between the TW's and the insulating alkyl molecules. This could explain why the observed switching behavior was independent of the functionalization of the molecules.

In conclusion, we find that functionalization of TW's has a stronger effect on the energetics of the monolayers than on the orbitals responsible for current transport, and a better understanding of the intermolecular interactions in such monolayers could hopefully be exploited in order to design molecular electronic devices with specific properties.

We thank J.-L. Mozos and P. Ordejon for help in implementing TRANSIESTA, J. Cornil for valuable discussions, and SNF (M.B.), STVF (K.S. and J.T.), NSERC (J.T.), EU SANEME Grant No. IST-1999-10323, Direktør Ib Henriksens fond, Danish Center for Scientific Computing (DCSC) for funding.
${ }^{1}$ C. Joachim, J.K. Gimzewski, and A. Aviram, Nature (London) 408, 541 (2000).

${ }^{2}$ J. Chen, M.A. Reed, A.M. Rawlett, and J.M. Tour, Science 286, 1550 (1999).

${ }^{3}$ J. Chen, W. Wang, M.A. Reed, A.M. Rawlett, D.W. Price, and J.M. Tour, Appl. Phys. Lett. 77, 1224 (2000).

${ }^{4}$ J. Chen and M.A. Reed, Chem. Phys. 281, 127 (2002).

${ }^{5}$ M.A. Reed, J. Chen, A.M. Rawlett, D.W. Price, and J.M. Tour, Appl. Phys. Lett. 78, 3735 (2001).

${ }^{6}$ J.M. Tour, M. Kozaki, and J.M. Seminario, J. Am. Chem. Soc. 120, 8486 (2001).

${ }^{7}$ Z.J. Donhauser, B.A. Mantooth, K.F. Kelly, L.A. Bumm, J.D. Monnell, J.J. Stapleton, J. David W. Price, A.M. Rawlett, D.L. Allara, J.M. Tour, and P.S. Weiss, Science 292, 2303 (2001).

${ }^{8}$ J.M. Seminario, A.G. Zacarias, and J.M. Tour, J. Am. Chem. Soc. 122, 3015 (2000).

${ }^{9}$ J.M. Seminario, A.G. Zacarias, and P.A. Derosa, J. Phys. Chem. A 105, 791 (2001).

${ }^{10}$ J. Cornil, Y. Karzazi, and J.L. Bredas, J. Am. Chem. Soc. 124(14), 3516 (2002).

${ }^{11}$ M. Brandbyge, J.-L. Mozos, P. Ordejon, J. Taylor, and K. Stokbro, Phys. Rev. B 65, 165401 (2002); see also www.transiesta.com

${ }^{12}$ In this study we have used both the local-density approximation (LDA), Refs. 25 and 26, and the generalized gradient approximation of Perdew, Burke, and Ernzerhof (PBE), Ref. 21. We find that the electrical properties are rather similar in PBE and LDA, while structural energies are slightly different. Unless otherwise stated, the presented results are obtained within LDA.

${ }^{13}$ M. DiVentra, S.G. Kim, S.T. Pantelides, and N.D. Lang, Phys. Rev. Lett. 86, 288 (2001).

${ }^{14}$ J. Taylor, M. Brandbyge, and K. Stokbro, Phys. Rev. Lett. 89, 138301 (2002)

${ }^{15}$ We use a double $\zeta$-polarization basis set for the organic mol- ecule, and single $\zeta$ for the gold $s, p$, and $d$ channel. Core electrons are described by Troullier-Martins pseudopotentials. The molecular geometry is obtained by first optimizing the geometry of the free molecule with $\mathrm{H}$ atoms attached to the sulfur atoms, then placing the molecule at the fcc positions with an Au-S distance of $2.45 \AA$, Ref. 27 , and finally relaxing the molecule in the sandwich structure with fixed Au atoms.

${ }^{16}$ S. Datta, Electronic Transport in Mesoscopic Systems (Cambridge University Press, New York, 1996).

${ }^{17}$ T.N. Todorov, J. Hoekstra, and A.P. Sutton, Philos. Mag. B 80, 421 (2000).

${ }^{18}$ Since the charging energy is inversely proportional to the distance between the molecules, we expect that doubling the distance between the molecules can at most double the charge on the molecules.

${ }^{19}$ E.G. Emberly and G. Kirczenow, Phys. Rev. B 58, 10911 (1998).

${ }^{20}$ J. Taylor, Ph.D. thesis, McGill University, 2000.

${ }^{21}$ J.P. Perdew, K. Burke, and M. Ernzerhof, Phys. Rev. Lett. 77, 3865 (1996).

${ }^{22}$ M. Böringer, K. Morgenstern, W.-D. Schneider, R. Berndt, F. Mauri, A.D. Vito, and R. Car, Phys. Rev. Lett. 83, 324 (1999).

${ }^{23}$ The statistically most likely geometry is that which minimizes the free energy: $E-\mu_{L} Q_{L}-\mu_{R} Q_{R}$, where $Q_{L}$ and $Q_{R}$ are the charges injected from the left and right electrodes (Ref. 17). The quantites $Q_{L}$ and $Q_{R}$ are, for technical reasons, very expensive to calculate. Furthermore, in order to conclusively determine which geometry has the lowest free energy, we should allow for relaxations of the atomic coordinates under the external bias.

${ }^{24}$ A.-A. Dhirani, R.W. Zehner, R.P. Hsung, P. Guyot-Sionnest, and L.R. Sita, J. Am. Chem. Soc. 118, 3319 (1996).

${ }^{25}$ J.P. Perdew and A. Zunger, Phys. Rev. B 23, 5048 (1981).

${ }^{26}$ D.M. Ceperley and B.J. Alder, Phys. Rev. Lett. 45, 566 (1980).

${ }^{27}$ W. Andreoni, A. Curioni, and H. Grönbeck, Int. J. Quantum Chem. 80, 598 (2000). 